\title{
Calcium Niobate Semiconductor Nanosheets as Catalysts for Photochemical Hydrogen Evolution from Water
}

\author{
Owen C. Compton, Elizabeth C. Carroll, Jin Y. Kim, Delmar S. Larsen,* and \\ Frank E. Osterloh* \\ Department of Chemistry, University of California, Davis, 1 Shields Ave, Davis, California 95616
}

Received: July 1, 2007; In Final Form: August 29, 2007

\begin{abstract}
We have studied the photochemical water splitting activity of individually dispersed, semiconductor nanosheets derived from the Dion-Jacobsen phase $\mathrm{HCa}_{2} \mathrm{Nb}_{3} \mathrm{O}_{10}$ by exfoliation with tetrabutylammonium hydroxide. The dimensions of the nanosheets are $0.001 \times 1.2 \times 1.2 \mu \mathrm{m}^{3}$ based on TEM measurements and crystallographic data, and their band gap is determined as $3.53 \mathrm{eV}$ based on the absorption band edge at $350 \mathrm{~nm}$. When an aqueous suspension of the nanosheets is irradiated with light from a Hg lamp, hydrogen is produced at rates of up to $2.28 \mu \mathrm{mol} / \mathrm{h}$ with a quantum efficiency $(\mathrm{QE})$ of $0.22 \%$. No oxygen is evolved. Derivatization of the nanosheets with Pt nanoparticles increases the hydrogen production rate to $78.37 \mu \mathrm{mol}(\mathrm{QE}=7.5 \%)$. Restacking of the nanosheets at low $\mathrm{pH}$ does not significantly affect the catalytic activity. Transient absorption measurements of the nanosheets reveal charge separation on a nanosecond time scale.
\end{abstract}

Predicted increases in global energy consumption and climate change driven by fossil fuel emissions have sparked a renewed interest in carbon-neutral energy sources. A recent analysis has shown that the conversion of solar energy into hydrogen is one of the most promising renewable energy technologies. ${ }^{1}$ Since the discovery of the first artificial photocatalytic water splitting system in $1971,2,3$ many inorganic materials have been identified as active catalysts for the photochemical reduction of water. ${ }^{4-8}$ However, limited light absorption in the visible, chemical instability of the catalyst materials, and short lifetimes of the excited states leading to incomplete charge separation continue to be key problems that prevent commercial applications of these catalysts. Some of these issues can be overcome with nanomaterials, whose large surface-to-volume ratio and short chargetransport pathways facilitate efficient charge transfer to water. This is well illustrated with layered group 4/5 metal oxides ( $\mathrm{Ti}$, $\mathrm{Nb}, \mathrm{Ta}$ ), whose exceptional quantum efficiencies for water reduction (up to $20 \%$ for $\mathrm{K}_{4} \mathrm{Nb}_{6} \mathrm{O}_{17}$ ) ${ }^{9}$ are commonly attributed to the large internal surface area of the material and improved charge transfer. ${ }^{10-15}$ For comparison, the best catalyst for overall water splitting, La-doped $\mathrm{NaTaO}_{3}$, has a quantum efficiency of $56 \% .{ }^{9}$ Here, we show for the first time that individually dispersed $\left[\mathrm{H}_{1-x} \mathrm{Ca}_{2} \mathrm{Nb}_{3} \mathrm{O}_{10}\right]^{x-}(x=0.15-0.20)$ nanosheets are able to split pure water under UV irradiation and evolve hydrogen, but no oxygen. We find that the photocatalytic activity of the nanosheets is strongly influenced by the solution $\mathrm{pH}$ and by the presence of Pt nanoparticles as cocatalysts. Transient absorption spectroscopy measurements provide information about the excited-state dynamics of the nanosheets.

Experimental details for the synthesis of the catalysts are given in the following. [(TBA $\left.)_{x} \mathrm{H}_{1-x} \mathrm{Ca}_{2} \mathrm{Nb}_{3} \mathrm{O}_{10}\right]$ nanosheets $(x=0.15-0.20, \mathrm{TBA}=$ tetrabutylammonium $)$ were synthesized

* To whom correspondence should be addressed. Fax: (+1) 530752 8995. Tel: (+1) 530752 6242. E-mail: fosterloh@ucdavis.edu (F.E.O.); dlarsen@ucdavis.edu (D.S.L.).

10.1021/jp0751155 CCC: $\$ 37.00$ according to literature procedures. ${ }^{16,18}$ Restacked $\mathrm{HCa}_{2} \mathrm{Nb}_{3} \mathrm{O}_{10}$ sheets were synthesized by adding dropwise a $0.5 \mathrm{M}$ solution of $\mathrm{HCl}$ to a dispersion of $100 \mathrm{mg}$ of $\mathrm{TBA}_{x}\left[\mathrm{H}_{1-x} \mathrm{Ca}_{2} \mathrm{Nb}_{3} \mathrm{O}_{10}\right]$ in $25 \mathrm{~mL}$ of water (purified by a Nanopure II system to a resistivity of $>18 \mathrm{M} \Omega$ ). The white precipitate was collected by centrifugation, washed twice with $50 \mathrm{~mL}$ aliquots of water, and suspended in $20 \mathrm{~mL}$ of water. Platinum nanoparticles were grown on the surface of $\mathrm{TBA}_{x}\left[\mathrm{H}_{1-x} \mathrm{Ca}_{2} \mathrm{Nb}_{3} \mathrm{O}_{10}\right]$ by irradiating nanosheets in $20 \%$ aqueous methanol containing 3 wt $\% \mathrm{H}_{2} \mathrm{PtCl}_{6}\left(\cdot 6 \mathrm{H}_{2} \mathrm{O}\right.$, Alfar Aesar). ${ }^{10}$ The product was collected by centrifugation, washed twice with $50 \mathrm{~mL}$ aliquots of water, and stored in $25 \mathrm{~mL}$ of water for future studies. For catalytic measurements, $100 \mathrm{mg}$ of the respective catalysts in $50 \mathrm{~mL}$ of pure water were placed into a quartz flask that was connected to a direct injection vacuum system. The flask was evacuated and purged with $\mathrm{Ar}$ gas three times to remove air and then irradiated with four $175 \mathrm{~W}$ commercial mercury lamps $(\lambda=290-600 \mathrm{~nm})$ while stirring. The quantum flux of this system was determined as $5.82 \times 10^{-7} \mathrm{~mol} / \mathrm{s}$ based on ferrioxalate actinometry. ${ }^{17}$ Gas samples were analyzed with a Varian gas chromatograph using Ar carrier gas, a 60/80A molecular sieve column, and a thermal conductivity detector. UV/vis spectra were collected using an Ocean Optics DH2000 light source and HR2000 CG-UVNIR spectrometer. Electron micrographs were obtained using a Philips CM120 transmission electron microscope. Transient absorption spectroscopy was carried out with a visible wavelength pump-probe spectrometer based on an amplified Ti: sapphire laser system (Spectra Physics Spitfire Pro + Tsunami, $40 \mathrm{fs}, 800 \mathrm{~nm}$ pulses, $1 \mathrm{kHz}$ ). A dispersed aqueous solution of $\mathrm{TBA}_{x}\left[\mathrm{H}_{1-x} \mathrm{Ca}_{2} \mathrm{Nb}_{3} \mathrm{O}_{10}\right]$ sheets flowing through a quartz cuvette was excited with $300 \mathrm{~nm}$ pulses generated as the second harmonic of a noncollinear optical parametric amplifier $(10 \mu \mathrm{J}$, $30 \mathrm{fs}$ pulses at $600 \mathrm{~nm}$ ). The probe was a femtosecond whitelight continuum $(450-750 \mathrm{~nm})$ variably delayed between $-10 \mathrm{ps}$ and $\sim 8 \mathrm{~ns}$. Changes in absorption were monitored on 


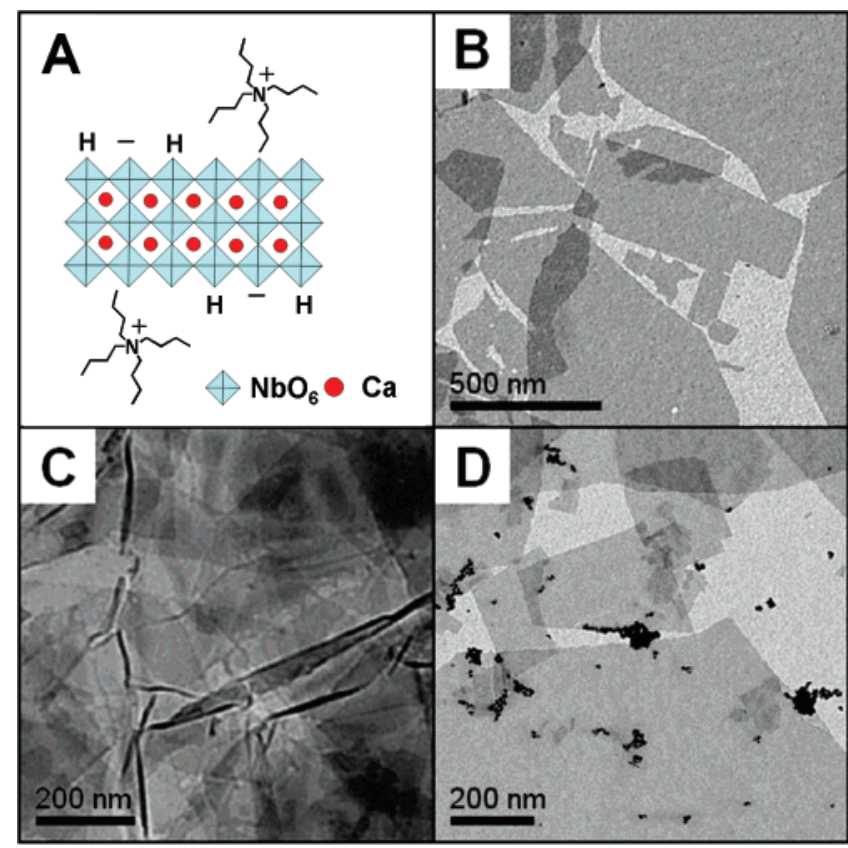

Figure 1. (A) Schematic structure of $\left(\mathrm{TBA}_{x}\right) \mathrm{H}_{1-x} \mathrm{Ca}_{2} \mathrm{Nb}_{3} \mathrm{O}_{10}$ nanosheets $(x=0.15-0.20)$. (B) Transmission electron micrograph (TEM) of exfoliated $\mathrm{TBA}_{x}\left[\mathrm{H}_{1-x} \mathrm{Ca}_{2} \mathrm{Nb}_{3} \mathrm{O}_{10}\right]$ nanosheets. (C) TEM of restacked $\mathrm{HCa}_{2} \mathrm{Nb}_{3} \mathrm{O}_{10}$. (D) TEM of $\mathrm{TBA}_{x}\left[\mathrm{H}_{1-x} \mathrm{Ca}_{2} \mathrm{Nb}_{3} \mathrm{O}_{10}\right]-\mathrm{Pt}$.

a shot-by-shot basis with a 256 pixel diode array. The pump source was optically chopped at $500 \mathrm{~Hz}$, and at each time delay, 1000 pumped/unpumped pairs of spectra were collected. All of the calcium niobate solutions were prepared to an optical density of $\sim 0.45 \pm 0.05$ at $300 \mathrm{~nm}$. A 250 fs instrument response function was determined by two-photon absorption in methanol.

Individual $\mathrm{TBA}_{x}\left[\mathrm{H}_{1-x} \mathrm{Ca}_{2} \mathrm{Nb}_{3} \mathrm{O}_{10}\right](x=0.15-0.20)$ semiconductor nanosheets could be obtained by treatment of the DionJacobsen phase $\mathrm{HCa}_{2} \mathrm{Nb}_{3} \mathrm{O}_{10}$ with $\mathrm{TBA}(\mathrm{OH}) .{ }^{18}$ The sheets consisted of three layers of corner-shared $\mathrm{NbO}_{6}$ octahedra with $\mathrm{Ca}^{2+}$ ions in the $\mathrm{O}$ voids (Figure 1A). As synthesized, the nanosheets were negatively charged with $15-20 \%$ of the protons replaced with tetrabutylammonium counterions. ${ }^{18}$ Under these conditions, the sheets occurred as individual particles with a flat morphology and edges that were mostly irregularly shaped and occasionally straight (Figure 1B). Edge lengths ranged from 0.2 to $1.8 \mu \mathrm{m}$, with a mean value of $1.2 \mu \mathrm{m}$. The thickness of the sheets was $1.16 \mathrm{~nm}$, from crystallographic data. ${ }^{16}$

Acidification of a nanosheet dispersion with dilute hydrochloric acid caused the nanosheets to stack up and precipitate from solution (eq 1). Transmission electron microscopy (Figure 1C) confirmed that this solid was comprised of irregularly stacked and folded nanosheets. No single sheets were observed.

$$
\begin{aligned}
\mathrm{TBA}_{x}\left[\mathrm{H}_{1-x} \mathrm{Ca}_{2} \mathrm{Nb}_{3} \mathrm{O}_{10}\right](x= & 0.15-0.20)+x \mathrm{HCl} \rightarrow \\
& {\left[\mathrm{HCa}_{2} \mathrm{Nb}_{3} \mathrm{O}_{10}\right]+x \mathrm{TBA}(\mathrm{Cl}) }
\end{aligned}
$$

Platinum nanoparticles were deposited onto individual nanosheets, as described above. TEM images of the product isolated by centrifugation revealed 7.2 $\pm 1.2 \mathrm{~nm}$ Pt nanoparticles deposited along the edges of the nanosheets (Figure 1D). The preferential Pt growth in these areas indicates that electrons accumulate at the edges of the nanosheets. It is also possible that $\mathrm{Pt}$ particles grew here because dangling $\mathrm{Nb}-\mathrm{O}$-bonds provided Pt nucleation sites that were absent from the surface of the sheets. The optical properties of the nanosheets are illustrated in Figure 2A. The nanosheet semiconductors absorb light in the ultraviolet region of the spectrum with an absorption
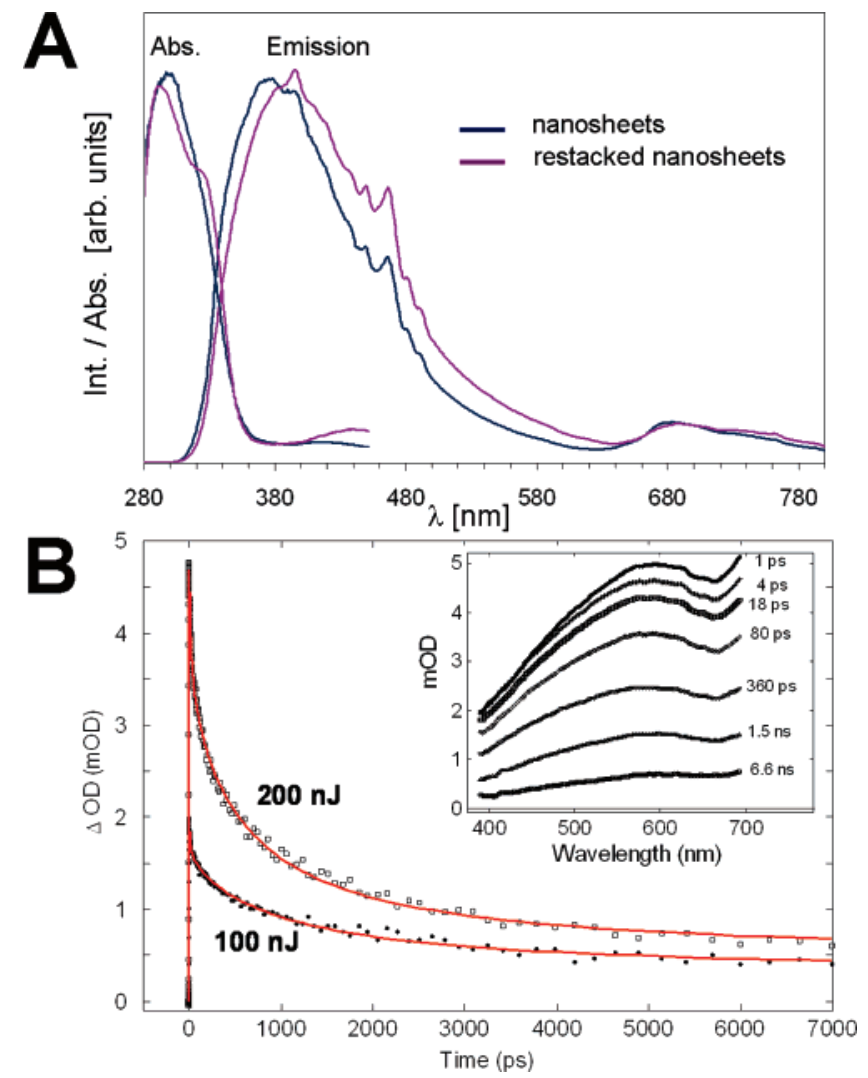

Figure 2. (A) Absorption (diffuse reflectance) and emission spectra of exfoliated nanosheets and restacked nanosheets. (B) Kinetics at $650 \mathrm{~nm}$ and transient absorption spectra (inset) of exfoliated nanosheets. The solid lines (red) represent a fit of the kinetics to eq 2. All spectra are for samples in aqueous suspension.

edge at $350 \mathrm{~nm}$, from which the band gap can be estimated as $3.53 \mathrm{eV}$. For comparison, bulk $\mathrm{KCa}_{2} \mathrm{Nb}_{3} \mathrm{O}_{10}$ has a band gap of $3.35 \mathrm{eV} .{ }^{19}$ The photoemission maximum of the nanosheets occurred at $381 \mathrm{~nm}(3.25 \mathrm{eV})$ and extended well into the visible, indicating that emission from surface trap states dominates the band-edge emission..$^{20}$ Upon restacking, the emission was shifted to $390 \mathrm{~nm}$, probably as a result of an increase in the density of surface defects.

Transient absorption spectroscopy was used to characterize the dynamics of photogenerated carriers at $\mathrm{pH}$ 10.5. Following $300 \mathrm{~nm}$ excitation, the dispersed niobate sheets exhibited a broad excited-state absorption with a maximum around $600 \mathrm{~nm}$ (Figure $2 \mathrm{~B}$, inset). This feature is similar to the trapped electron spectrum observed in other semiconductor nanoparticles..$^{21,22}$ The rise of the signal showed that carrier trapping occurred within $1 \mathrm{ps}$. The transient spectrum decay is nearly uniform. Kinetics observed at $\lambda_{\text {probe }}=650 \mathrm{~nm}$ are shown in Figure 2B.

The kinetics depends on laser fluence. This behavior is consistent with a second-order recombination process in which the rate of decay depends on the number of electron-hole pairs present on each nanosheet. However, the kinetics cannot be represented with a single decay constant. To estimate the time scales of charge separation, the kinetics was fit to a secondorder equation

$$
\Delta \mathrm{OD}(\mathrm{t})=\left[k_{1} t+1 / P_{1}\right]^{-1}+\left[k_{2} t+1 / P_{2}\right]^{-1}+C
$$

where $P_{1}$ and $P_{2}$ are the photogenerated electronic populations decaying at rates $k_{1}$ and $k_{2}$, respectively, and $C$ is a baseline representing a long-lived component. The resulting time constants were $k_{1}^{-1}=28 \pm 1 \mathrm{ps}$ and $\mathrm{k}_{2}^{-1}=1.75 \pm 0.05 \mathrm{~ns}$. The 

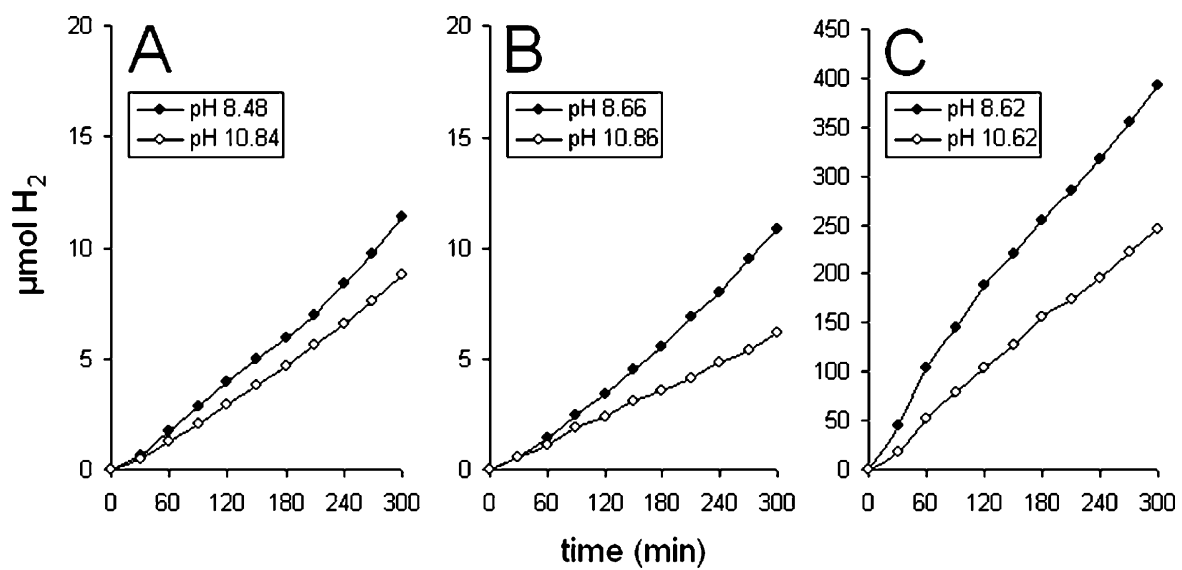

Figure 3. Time-dependent hydrogen evolution of (A) exfoliated $\mathrm{TBA}_{x}\left[\mathrm{H}_{1-x} \mathrm{Ca}_{2} \mathrm{Nb}_{3} \mathrm{O}_{10}\right]$, (B) restacked $\mathrm{HCa}_{2} \mathrm{Nb}_{3} \mathrm{O}_{10}$, and $(\mathrm{C}) \mathrm{Pt}_{-10 a d e d}$ $\mathrm{TBA}_{x}\left[\mathrm{H}_{1-x} \mathrm{Ca}_{2} \mathrm{Nb}_{3} \mathrm{O}_{10}\right]$. Results are for $100 \mathrm{mg}$ of catalyst dispersed in $50 \mathrm{~mL}$ of water at the indicated pH values in a $100 \mathrm{~mL}$ quartz flask under irradiation with a $750 \mathrm{~W} \mathrm{Hg}$ illumination system.

remaining fit parameters for $\lambda_{\text {probe }}=650 \mathrm{~nm}$ were $C=0.44$ and $P_{2} / P_{1} \sim 2$.

Previous transient absorption studies on single crystalline thin film $\mathrm{K}_{4} \mathrm{Nb}_{6} \mathrm{O}_{17}$ reported a single 150 ps second-order recombination lifetime, ${ }^{22}$ which falls between the rates determined in our exfoliated sheets. The lifetime $k_{1}^{-1}$ is comparable to the second-order lifetime reported in colloidal $\mathrm{TiO}_{2}$, where nongeminate electron-hole recombination and higher-order nonradiative relaxation processes dominate the trapped carrier dynamics. ${ }^{23}$ A recent study of nanocrystalline $\mathrm{TiO}_{2}$ under weak excitation (one electron-hole pair per particle) found a much longer electronic relaxation time scale $(500 \mathrm{ps}){ }^{24}$ The rate $k_{2}$ in the exfoliated niobate sheets is more comparable to weakexcitation conditions. Although a more complex model of recombination dynamics is necessary, ${ }^{21,25,26}$ the longer lifetime found by preliminary analysis indicates long-lived charge separation, which is necessary for catalytic activity.

The results of photocatalytic measurements for a nanosheet dispersion at $\mathrm{pH}=10.8$ are shown in Figure 3A. Hydrogen evolution occurs at a steady rate $(1.75 \mu \mathrm{mol} / \mathrm{h})$ but with a low quantum efficiency $(\mathrm{QE})$ of $0.17 \%\left(\mathrm{QE}=2 \cdot\left[\mathrm{H}_{2}\right] / I\right.$, with $\left[\mathrm{H}_{2}\right]$ as a rate of $\mathrm{H}_{2}$ production [mol/s] and $I$ as the quantum flux [mol/s]). After $24 \mathrm{~h}$, the $\mathrm{H}_{2}$ evolution rate is unchanged, and TEM data recorded on samples after irradiation reveal no noticeable change of the nanosheets (Supporting information). This suggests that $\mathrm{H}_{2}$ evolution does not proceed by photochemical degradation of the semiconductor. The efficiency of the nanosheet catalyst can be enhanced slightly $(2.28 \mu \mathrm{mol} / \mathrm{h}$, $\mathrm{QE}=0.22 \%$ ) by lowering the $\mathrm{pH}$ to 8.5 . This indicates that the $\mathrm{pH}$ dependence of the semiconductor flat-band potential is overcompensated by a more favorable reduction potential of $\mathrm{H}_{3} \mathrm{O}^{+}$at lower $\mathrm{pH}(-0.50 \mathrm{~V}$ at $\mathrm{pH}=8.5 \mathrm{vs}-0.64 \mathrm{~V}$ at $\mathrm{pH}=$ 10.8).

For comparison purposes, the catalytic activity of restacked $\mathrm{HCa}_{2} \mathrm{Nb}_{3} \mathrm{O}_{10}$ was also determined. At $\mathrm{pH}=10.9$, a dispersion of this material produced hydrogen at $1.23 \mu \mathrm{mol} / \mathrm{h}$ with a $\mathrm{QE}$ of $0.12 \%$ (Figure 2B). Again, the activity of the stacked sheets is $\mathrm{pH}$-dependent. After acidification of the dispersion with $\mathrm{HCl}$ to $\mathrm{pH}=8.7$, enhanced $\mathrm{H}_{2}$ production took place at a rate of $2.17 \mu \mathrm{mol} / \mathrm{h}$ (QE of $0.21 \%$ ). This value is only slightly below the value for individual sheets at the same $\mathrm{pH}=8.5$. This shows that the catalytically active area of the nanosheets is not reduced by stacking. The presence of solvent-accessible interlayer space is well documented for the layered solids $\mathrm{MCa}_{2} \mathrm{Nb}_{3} \mathrm{O}_{10}(\mathrm{M}=$ $\mathrm{H}, \mathrm{Na}, \mathrm{K}, \mathrm{Rb}, \mathrm{Cs}){ }^{16,19}$

It is known that the addition of Pt cocatalysts to $\mathrm{K}(\mathrm{H}) \mathrm{Ca}_{2}$ $\mathrm{Nb}_{3} \mathrm{O}_{10}$ can increase the catalytic activity of methanol dehy-

TABLE 1: Hydrogen Evolution Data

\begin{tabular}{lcccccc}
\hline & \multicolumn{2}{c}{ nanosheets } & \multicolumn{2}{c}{ restacked sheets } & \multicolumn{2}{c}{ Pt-loaded nanosheets } \\
\hline $\mathrm{pH}$ & 8.48 & 10.8 & 8.66 & 10.9 & 8.62 & 10.6 \\
$\mathrm{H}_{2}[\mu \mathrm{mol}]$ after 5 h & 11.4 & 8.77 & 10.9 & 6.17 & 392 & 246 \\
$\mathrm{H}_{2}$ rate $[\mu \mathrm{mol} / \mathrm{h}]$ & 2.28 & 1.75 & 2.17 & 1.23 & 78.4 & 49.2 \\
$\mathrm{QE}[\%]$ & 0.22 & 0.17 & 0.21 & 0.12 & 7.48 & 4.69
\end{tabular}

drogenation. ${ }^{10,19,27}$ We observe an analogous effect on the water splitting activityofindividually dispersedPt-decorated $\left[\mathrm{H}_{1-x} \mathrm{Ca}_{2} \mathrm{Nb}_{3} \mathrm{O}_{10}\right]^{x-}$ nanosheets. Suspended in water at $\mathrm{pH}=10.6$, the nanosheet composite produces $\mathrm{H}_{2}$ at a rate of $49.15 \mu \mathrm{mol} / \mathrm{h}(\mathrm{QE}=4.7 \%)$, a nearly 30-fold increase over the Pt-free nanosheets at the same $\mathrm{pH}$. By lowering the $\mathrm{pH}$ to 8.62 , the activity nearly doubles $(78.4 \mu \mathrm{mol} / \mathrm{h}, \mathrm{QE}=7.48 \%)$, probably as a consequence of the more-favorable proton reduction potential.

Remarkably, $\mathrm{O}_{2}$ could not be detected in any of the experiments. The inability of layered niobates to produce oxygen in the absence of oxidizing agents or cocatalysts is well documented. ${ }^{19,28-32}$ We attribute this observation to the possible adsorption of water oxidation products to the nanosheets. In order to examine the possibility that TBA acts as a sacrificial electron donor in the present system, we also conducted a $24 \mathrm{~h}$ irradiation experiment (Supporting information) but found no decrease in the $\mathrm{H}_{2}$ evolution rate. Furthermore, we used combustion analysis to determine the TBA content in dispersed and restacked nanosheets (Supporting Information). In agreement with eq 1, the volatiles content (water and TBA) is markedly lower in the restacked sheet sample (4.2 mass \%) than that in the dispersed sheet sample (12.3 mass \%). Despite this difference, both samples produce $\mathrm{H}_{2}$ at nearly identical rates (Table 1), indicating that TBA does not serve as a sacrificial electron donor. Hydrogen evolution rates of other semiconductors have been previously found to vary markedly with the concentration of sacrificial electron donors. ${ }^{33,34}$

In summary, we have carried out the first study on the photocatalytic water splitting reaction with dispersed semiconductor nanosheets. Under irradiation with UV light, tetrabutylammonium-stabilized $\left[\mathrm{H}_{1-x} \mathrm{Ca}_{2} \mathrm{Nb}_{3} \mathrm{O}_{10}\right]^{x-}$ nanosheets produce hydrogen from pure water, but no oxygen. The catalytic activity is enhanced at low $\mathrm{pH}$ and with Pt nanoparticle cocatalysts. It is not significantly diminished upon aggregation. Optical characterization of the nanosheets supports a long-lived charge separation following UV excitation. Additional studies aimed at identifying oxidation products and optimizing catalytic activity are underway.

Acknowledgment. This work was supported by an Energy Innovation Startup Grant of the California Energy Commission. 
We thank Professor Susan Kauzlarich for assistance with hightemperature solid-state reactions.

Supporting Information Available: TEM images of nanosheets, $24 \mathrm{~h}$ hydrogen evolution data, visible spectrum of the mercury lamp, and combustion analysis results. This material is available free of charge via the Internet at http://pubs.acs.org.

\section{References and Notes}

(1) Lewis, N. S.; Nocera, D. G. Proc. Natl. Acad. Sci. U.S.A. 2006 103,15729 .

(2) Fujishima, A.; Honda, K. Bull. Chem. Soc. Jpn. 1971, 44, 1148.

(3) Fujishima, A; Honda, K. Nature 1972, 238, 37.

(4) Bard, A. J.; Fox, M. A. Acc. Chem. Res. 1995, 28, 141.

(5) Kamat, P. V.; Meisel, D. C. R. Chim. 2003, 6, 999.

(6) Gurevich, Y. Y.; Pleskov, Y. V. Semiconductor Photoelectrochemistry; Consultants Bureau: New York, 1986; p 422.

(7) Ohashi, K.; McCann, J.; Bockris, J. O. M. Nature 1977, 266, 610

(8) Nowotny, J.; Sorrell, C. C.; Bak, T.; Sheppard, L. R. Sol. Energy 2005, 78, 593 .

(9) Kato, H.; Asakura, K.; Kudo, A. J. Am. Chem. Soc. 2003, 125, 3082.

(10) Ebina, Y.; Sasaki, T.; Harada, M.; Watanabe, M. Chem. Mater. 2002, 14, 4390

(11) Iwase, A.; Kato, H.; Kudo, A. Catal. Lett. 2006, 108, 6.

(12) Kim, Y. I.; Atherton, S. J.; Brigham, E. S.; Mallouk, T. E. J. Phys. Chem. 1993, 97, 11802.

(13) Kim, H. G.; Becker, O. S.; Jang, J. S.; Ji, S. M.; Borse, P. H.; Lee, J. S. J. Solid State Chem. 2006, 179, 1214.

(14) Kim, H. G.; Hwang, D. W.; Kim, J.; Kim, Y. G.; Lee, J. S. Chem. Commun. 1999, 1077.

(15) Domen, K. Water Photolysis by Layered Compounds. In Photocatalysis Science and Technology; Kaneko, M., Okura, I., Eds.; Springer:
New York, 2002; pp 261-278.

(16) Jacobsen, A. J.; Johnson, J. W.; Lewandowski, J. T. Inorg. Chem. 1985, 24, 3727.

(17) Kuhn, H. J.; Braslavsky, S. E.; Schmidt, R. Pure Appl. Chem. 2004, $76,2105$.

(18) Schaak, R. E.; Mallouk, T. E. Chem. Mater. 2000, 12, 2513.

(19) Domen, K.; Yoshimura, J.; Sekine, T.; Tanaka, A.; Onishi, T. Catal. Lett. 1990, 4, 339 .

(20) Burda, C.; El-Sayed, M. A. Pure Appl. Chem. 2000, 72, 165.

(21) Rothenberger, G.; Moser, J.; Gratzel, M.; Serpone, N.; Sharma, D. K. J. Am. Chem. Soc. 1985, 107, 8054

(22) Furube, A.; Shiozawa, T.; Ishikawa, A.; Wada, A.; Domen, K.; Hirose, C. J. Phys. Chem. B 2002, 106, 3065.

(23) Colombo, D. P.; Roussel, K. A.; Saeh, J.; Skinner, D. E.; Cavaleri, J. J.; Bowman, R. M. Chem. Phys. Lett. 1995, 232, 207.

(24) Tamaki, Y.; Furube, A.; Murai, M.; Hara, K.; Katoh, R.; Tachiya, M. Phys. Chem. Chem. Phys. 2007, 9, 1453

(25) Grela, M. A.; Colussi, A. J. J. Phys. Chem. 1996, 100, 18214.

(26) Beadie, G.; Sauvain, E.; Lawandy, N. M. Solid State Commun. 1995, 94,709 .

(27) Ebina, Y.; Tanaka, A.; Kondo, J. N.; Domen, K. Chem. Mater. 1996, 8, 2534

(28) Takata, T.; Tanaka, A.; Hara, M.; Kondo, J. N.; Domen, K. Catal. Today 1998, 44, 17.

(29) Sayama, K.; Yase, K.; Arakawa, H.; Asakura, K.; Tanaka, A.; Domen, K.; Onishi, T. J. Photochem. Photobiol., A 1998, 114, 125.

(30) Sayama, K.; Tanaka, A.; Domen, K.; Maruya, K.; Onishi, T. Catal. Lett. 1990, 4, 217.

(31) Kudo, A.; Kato, H.; Nakagawa, S. J. Phys. Chem. B 2000, 104 571.

(32) Ebina, Y.; Sakai, N.; Sasaki, T. J. Phys. Chem. B 2005, 109, 17212.

(33) Buhler, N.; Meier, K.; Reber, J. F. J. Phys. Chem. 1984, 88, 3261.

(34) Reber, J. F.; Meier, K. J. Phys. Chem. 1984, 88, 5903. 